\title{
Capacidade Funcional e Qualidade de Vida de Idosas Usuárias das Academias da Terceira Idade e não Praticantes de Exercício Físico
}

\author{
Daniel Vicentini Oliveira, ${ }^{1}$ Paolo Marcello Cunha, ${ }^{2}$ Gabriel Lucas Morais Freire, ${ }^{3}$ \\ José Roberto Andrade do Nascimento Júnior, ${ }^{4}$ Sônia Maria Marques Gomes Bertolini ${ }^{5}$
}

\begin{abstract}
RESUMO
O objetivo do presente estudo foi avaliar a capacidade funcional e qualidade de vida de mulheres idosas que praticavam exercícios nas Academias da Terceira Idade (ATIs) e idosas não praticantes de nenhum tipo de exercício e analisar a correlação dos parâmetros da capacidade funcional e qualidade de vida desses sujeitos. Trata-se de um estudo transversal, realizado com 80 idosas. A amostra foi dividida em grupo não praticante de exercício físico (G1) e usurário das ATIs (G2). Foi utilizado um questionário sociodemográfico e de saúde, o WHOQOL-BREF e o WHOQOL-OLD. A capacidade funcional foi avaliada por meio dos testes do Protocolo de Avaliação Funcional do Grupo de Desenvolvimento Latino-Americano para Maturidade (GDLAM). A análise dos dados foi feita por meio dos testes Kolmogorov-Smirnov, Qui-quadrado de Pearson ( $\mathrm{X}^{2}$ ) e correlação de Spearman. Considerou-se um nível de significância de $p<0,05$. As idosas do $G 2$ apresentaram maior escore na faceta de qualidade de vida de Participação Social $(p=0,004)$. As integrantes do $\mathrm{G} 2$ realizaram o teste de levantar da cadeira e mover-se pela casa em menor tempo $(p=0,001)$, enquanto as idosas do G1 fizeram em menor tempo o teste de vestir e tirar a camiseta $(p=0,001)$. Os melhores escores obtidos no item levantar-se da cadeira e mover-se pela casa indicam importantes contribuições possíveis das ATI para a dimensão física das condições de saúde dos idosos, além do benefício na socialização verificada pela percepção da qualidade de vida social dessa população.
\end{abstract}

Palavras-chave: Envelhecimento. Atividade motora. Gerontologia.

\section{FUNCTIONAL CAPACITY AND QUALITY OF LIFE IN OLDER ADULTS OF FITNESS ZONE} AND NOT PHYSICAL EXERCISES PRACTICE

\begin{abstract}
The aim of this study was to analyze the functional capacity and quality of life of elderly women who exercise in the fitness zone (ATI) and elderly women who do not practice any type of exercise and to analyze the correlation of the parameters of the functional capacity and quality of life. life of these subjects. This is a cross-sectional study conducted with 80 elderly women. The sample was divided into non-physical exercise group (G1) and ATI user (G2). A sociodemographic and health questionnaire, WHOQOL-BREF and WHOQOL-OLD were used. Functional capacity was assessed by testing the Functional Assessment Protocol of the Latin American Development Group for Maturity (GDLAM). Data analysis was performed using the Kolmogorov-Smirnov, Pearson Chi-square (X2) and Spearman correlation tests. A significance level of $p<0.05$ was considered. The elderly women from $\mathrm{G} 2$ had the highest score in the quality of life facet of Social Participation $(p=0.004)$. The elderly women from $G 2$ underwent the chair lift test and move around the house in a shorter time $(p=0.001)$, while the elderly from G1 performed the dress and take off shirt test $(p=0.001)$. The best scores obtained in the item rising from the chair and moving around the house indicate important possible contributions of the ATI to the physical dimension of health conditions of the elderly, in addition beyond the benefit in socialization, verified by the perception of social quality of life of this population.

Keywords: Aging. Motor acitivy. Gerontology.
\end{abstract}

RECEBIDO EM: 6/9/2019

MODIFICAÇÕES REQUERIDAS EM: 24/10/2019

ACEITO EM: 8/11/2019

\footnotetext{
${ }^{1}$ Doutor em Gerontologia. Pós-doutorando em Promoção da saúde. Centro Universitário de Maringá (Unicesumar).d.vicentini@hotmail.com

Doutorando em Educação Física. Universidade Estadual de Londrina (UEL).pcunha88@hotmail.com

Mestrando em Educação Física. Universidade Federal do Vale do São Francisco (Univasf). bi88el@gmail.com

${ }^{4}$ Doutor em Educação física. Docente no Programa de Pós-Graduação Stricto Sensu em Educação física. Universidade Federal do Vale do São Francisco (Univasf). jroberto.jrs01@gmail.com

Doutora em Ciências Morfológicas. Docente e coordenadora do Programa de Pós-Graduação Stricto Sensu em Promoção da saúde. Centro Universitário de Maringá (Unicesumar). smmgbertolini@yahoo.com.br
} 


\section{INTRODUÇÃO}

A expectativa de vida aumentou consideravelmente ao logo do tempo, carregando mudanças demográficas pelo mundo (BARBER; FORSTER; BIRCH, 2015). Segundo a Organização Mundial da Saúde (OMS, 2015), estima-se que até 2050 cerca de $20 \%$ da população global será de idosos. Com essa expectativa de vida aumentada cresce o interesse em fatores de envelhecimento bem-sucedidos para obter vida saudável na velhice (PINTO; FONTAINE; NERI, 2016).

Como se sabe, durante o processo de envelhecimento é observada uma série de modificações morfológicas, metabólicas, psicológicas, funcionais, que podem impactar negativamente a qualidade de vida da população idosa (FREITAS et al., 2016; GUTIÉRREZ; CALATAYUD; TOMÁS, 2018). Essas alterações, em especial a redução da capacidade funcional, têm grande importância nas atividades básica da vida diária (ABVD), como sentar, levantar, vestir-se e nas atividades instrumentais da vida diária (AIVD), que são tarefas mais complexas, como utilizar meio de transporte, participação social, realizar compras (FARÍAS-ANTÚNEZ et al., 2018).

Pesquisas recentes, entretanto, afirmam que um dos mais importantes produtos não farmacêuticos para o envelhecimento saudável é o exercício físico (RIBEIRO et al., 2015; SANTOS et al., 2016; MÜLLER et al., 2016; VAN ALPHEN; HORTOBAGYI; VAN HEUVELEN, 2016), uma vez que sua prática está associada à melhora dos aspectos físicos, fisiológicos (SIQUEIRA et al., 2017; AGOSTINI et al., 2018) e psicológicos (SILVA et al., 2016; CHUNG et al., 2017).

Com isso, políticas públicas para o desenvolvimento de programas de exercícios para a população idosa surgem como uma expectativa de longevidade e devem ser mantidos e reforçados, com o objetivo de garantir condições de saúde, oportunidade, participação e melhorar a qualidade de vida do idoso (DANTAS; VALE, 2004). Qualidade de vida que é uma sistematização multidimensional relacionada à saúde, à funcionalidade física e cognitiva, à atividade, à produtividade e à capacidade de regulação emocional, bem como ao bem-estar subjetivo e econômico e aos recursos ecológicos e sociais compatíveis com as necessidades de um idoso (OLIVEIRA et al., 2017).

Sabendo disso, as Academias da Terceira Idade (ATIs) podem ser uma boa opção para a prática de exercício físico para o idoso, visto que é uma atividade gratuita, além de favorecer um bom ambiente de convívio social dessa população (GONÇALVES et al., 2015). Não se tem, entretanto, bem estabelecido se melhores condições funcionais estão relacionadas com melhores percepções de qualidade de vida des- se segmento da população. E é verificar esta relação entre qualidade de vida e capacidade funcional que visamos neste estudo.

Nesse sentido, o objetivo do presente trabalho foi analisar a capacidade funcional e qualidade de vida de mulheres idosas que praticavam exercícios nas ATI e idosas não praticantes de nenhum tipo de exercício físico e avaliar a correlação dos parâmetros da capacidade funcional e qualidade de vida desses sujeitos. Formulou-se a hipótese de que praticantes de exercícios físicos nessas ATIs teriam maiores escores de capacidade física e, consequentemente, melhores percepções de qualidade de vida do que idosas que não praticam exercícios físicos.

\section{MATERIAIS E MÉTODOS}

Trata-se de uma pesquisa quantitativa, observacional e transversal, aprovada pelo Comitê de Ética em Pesquisa (CEP) com Seres Humanos do Centro Universitário de Maringá por meio do parecer número 217.895/2013.

\section{Participantes}

Participaram deste estudo 80 idosas, divididas em dois grupos de 40: idosas não praticantes de exercício físico (G1) e idosas usuárias das ATIs (G2). A amostra foi escolhida de forma aleatória e por conveniência e as voluntárias foram informadas quanto à justificativa, objetivos e procedimentos a serem realizados.

Foram incluídas no estudo idosas (60 anos ou mais) usuárias das ATI, ou não praticantes de exercício físico, residentes no município de Maringá, Paraná. Foram excluídas idosas que apresentavam alterações neurológicas (com sinais e sintomas motores e psíquicos) limitantes para responder os questionários, assim como aquelas com possíveis déficits cognitivos, avaliados pelo Mini Exame do Estado Mental (Meem). O Meem é composto por questões agrupadas em sete categorias: orientação temporal, orientação espacial, registro de três palavras, atenção e cálculo, recordação das três palavras, linguagem e capacidade visoconstrutiva (FOLSTEIN; FOLSTEIN; MCHUGH, 1975). As notas de corte utilizadas para exclusão pelo Meem foram: 17 para os analfabetos; 22 para idosos com escolaridade entre 1 e 4 anos; 24 para os com escolaridade entre 5 e 8 anos e 26 para os que tiverem 9 ou mais anos de escolaridade. Correspondem à média obtida por esses autores para cada faixa de escolaridade, menos um desvio padrão (BRUCKI et al., 2003). Idosos classificados abaixo do ponto de corte específico para sua escolaridade foram excluídos. 


\section{Instrumentos}

Primeiramente foi aplicado um questionário semiestruturado pelos próprios autores com questões relacionas à idade, faixa etária (60 a 69 anos; 70 anos ou mais), estado civil (casada; não casada), renda mensal em salários mínimos de referência no ano de 2013 (1 a 2 salários mínimos; mais de 2 salários mínimos) e situação ocupacional (ativo; não ativo).

A avaliação da qualidade de vida foi realizada por meio dos questionários WHOQOL-BREF e WHQOOL-OLD. O WHOQOL-BREF é composto por 26 questões, das quais 2 referem-se à percepção individual da qualidade de vida e da percepção de saúde e as demais são subdivididas em quatro domínios: físico, psicológico, relações sociais e meio ambiente. O escore varia de 4 a 20, observando-se que quanto mais próximo de 20 melhor a qualidade de vida no domínio avaliado (FLECK et al., 2000). Já o WHOQOL-OLD consiste de 24 facetas, atribuídos a 6 domínios: funcionamento dos sentidos, autonomia, atividades passadas, presentes e futuras, participação social, morte e morrer e intimidade. Cada item é composto por quatro questões. Foi escolhido e utilizado o escore transformado, que é calculado a partir da conversão do escore bruto (apresentado a partir da somatória de cada questão, variando o resultando entre 4 e 20) para uma escala de 0 a 100 . Quanto mais próximo de 100 , melhor a qualidade de vida no domínio avaliado. O escore total do WHOQOL-OLD pode ser calculado por meio da soma das 24 questões do instrumento e é obtido o escore bruto, variante entre 24 e 120 . A conversão do escore bruto para uma escala de 0 a 100 representa o escore transformado (FLECK; CHACHAMOVICH; TRENTINI, 2006).

A capacidade funcional foi avaliada por meio dos testes do Protocolo de Avaliação Funcional do Grupo de Desenvolvimento Latino-Americano para Maturidade (GDLAM): caminhar 10 metros $\mathrm{C} 10 \mathrm{~m}$ ), levantar-se da posição sentada (LPS), levantar-se da posição de decúbito ventral (LPDV), levantar-se da cadeira e mover-se pela casa (LCMC) (DANTAS; VALE, 2004) e vestir e tirar uma camiseta (VTC) (VALE et al., 2008).

Todos os testes foram utilizados para calcular o índice de GDLAM (IG), e os tempos foram calculados em segundos. Quanto menor a pontuação obtida, maior o nível de capacidade funcional. O GDLAM foi calculado da seguinte forma:

$I G=[(C 10 m+L P S+L P D V+V T C) \times 2]+L C M C$

\section{Procedimentos}

A coleta dos dados foi realizada pelos próprios pesquisadores, juntamente com uma equipe de profissionais colaboradores, no período de abril a agosto de 2013, em cinco ATIs (Parque do Ingá, Vila Olímpica, Parque das Grevilhas, Bosque II e Parigot de Souza). As ATIs foram escolhidas de forma intencional e por conveniência, assim como pela maior prevalência de idosos usuários dos locais, segundo informações coletadas com a Secretaria de Esportes e Lazer do município. As idosas não praticantes de exercício físico foram abordadas em suas próprias residências, nos mesmos bairros onde se encontravam as ATIs citadas anteriormente. As participantes que aceitaram participar da pesquisa assinaram o Termo de Consentimento Livre e Esclarecido (TCLE).

\section{Análise dos Dados}

A análise dos dados foi realizada por meio do Software SPSS 22.0, utilizando-se uma abordagem de estatística descritiva e inferencial. Foi utilizada frequência e percentual como medidas descritivas para as variáveis categóricas. Para as variáveis numéricas, inicialmente foi verificada a normalidade dos dados por meio do teste Kolmogorov-Smirnov. Como os dados não apresentaram distribuição normal, foram utilizadas Mediana (Md) e Quartis (Q1; Q3) para a caracterização dos resultados. Na comparação entre os grupos (Não praticantes e ATI) foi utilizado o teste "U" de Mann-Whitney. O teste de Qui-quadrado de Pearson $\left(X^{2}\right)$ foi utilizado para se observar as diferenças nas proporções das variáveis sociodemográficas entre os dois grupos. Para verificar a correlação dos testes de capacidade funcional com os domínios e facetas de qualidade de vida, efetuou-se o coeficiente de correlação de Spearman. Considerou-se um nível de significância de $p<0,05$.

\section{Resultados}

Ao analisar a prevalência do perfil sociodemográfico das idosas de ambos os grupos (Tabela 1), não houve diferença significativa $(p>0,05)$ nas proporções das variáveis sociodemográficas, indicando que os mesmos apresentam perfis semelhantes quanto às referidas variáveis. 
Tabela 1 - Perfil sociodemográfico das idosas usuárias das ATIs e das idosas não praticantes de exercício físico

\begin{tabular}{|c|c|c|c|c|}
\hline \multirow{3}{*}{ VARIÁVEIS } & \multicolumn{2}{|c|}{ Grupos } & \multirow{3}{*}{$\mathrm{X}^{2}$} & \multirow{3}{*}{$\mathbf{P}$} \\
\hline & G1 & G2 & & \\
\hline & $f(\%)$ & $f(\%)$ & & \\
\hline \multicolumn{5}{|l|}{ Faixa etária } \\
\hline 60 a 69 anos & $37(54,4)$ & $31(45,6)$ & \multirow{2}{*}{3,529} & \multirow{2}{*}{0,060} \\
\hline 70 anos ou mais & $3(25,0)$ & $9(75,0)$ & & \\
\hline \multicolumn{5}{|l|}{ Estado civil } \\
\hline Casada & $19(46,3)$ & $22(53,7)$ & \multirow{2}{*}{0,450} & \multirow{2}{*}{0,502} \\
\hline Não casada & $21(53,8)$ & $18(46,2)$ & & \\
\hline \multicolumn{5}{|l|}{ Renda Mensal ${ }^{a}$} \\
\hline 1 a 2 salários mínimos & $21(45,7)$ & $25(54,3)$ & \multirow{2}{*}{0,818} & \multirow{2}{*}{0,366} \\
\hline Mais de 2 salários mínimos & $19(55,9)$ & $15(44,1)$ & & \\
\hline \multicolumn{5}{|l|}{ Situação Ocupacional } \\
\hline Ativo & $17(48,6)$ & $18(51,4)$ & \multirow{2}{*}{0,051} & \multirow{2}{*}{0,822} \\
\hline Inativo & $23(51,1)$ & $22(48,9)$ & & \\
\hline
\end{tabular}

*Associação significativa - $p<0,05$ : Teste de Qui-quadrado para proporções. a) Salário mínimo em 2015: R\$788,06 (US\$ 308,87).

ATI: Academias da Terceira idade; G1: idosas não praticantes de exercício físico; G2: idosas usuárias das ATIs.

Quando se comparou os domínios e facetas de qualidade de vida entre os grupos (Tabela 2), verificou-se diferença significativa apenas na faceta de Participação Social $(p=0,004)$, evidenciando que as idosas do G2 apresentaram maior escore.

Tabela 2 - Comparação dos domínios e facetas de qualidade de vida das idosas não praticantes de exercício físico (G1) e das idosas usuárias das Academias da Terceira Idade (G2)

\begin{tabular}{lccc}
\hline \multirow{2}{*}{ VARIÁVEIS } & G1 & G2 & \multirow{2}{*}{$\boldsymbol{P}$} \\
\cline { 2 - 3 } & Md (Q1;Q3) & Md (Q1;Q3) & \\
\cline { 1 - 3 } Domínios de Qualidade de vida (WHOQOL Bref) & \\
Domínio 1 - Físico & $15,4(13,7 ; 16,6)$ & $15,4(13,8 ; 17,7)$ & 0,489 \\
Domínio 2 - Psicológico & $15,3(14,7 ; 16,7)$ & $16,0(14,7 ; 17,3)$ & 0,194 \\
Domínio 3 - Relações sociais & $14,7(13,3 ; 16,0)$ & $15,3(13,3 ; 17,3)$ & 0,419 \\
Domínio 4 - Meio ambiente & $14,5(12,0 ; 15,5)$ & $14,0(12,5 ; 16,0)$ & 0,678 \\
Domínio 5 - Autoavaliação & $16,0(12,5 ; 17,5)$ & $16,0(14,0 ; 16,0)$ & 0,925 \\
Facetas de Qualidade de vida (WHOQOL Old) & & \\
Faceta 1-Funcionamento DS & $17,0(15,0 ; 17,0)$ & $15,5 ; 12,3 ; 17,8)$ & 0,131 \\
Faceta 2 - Autonomia & $15,0(12,0 ; 15,0)$ & $14,0(12,0 ; 15,0)$ & 0,218 \\
Faceta 3 - Atividades PPF & $15,0(13,0 ; 16,0)$ & $15,0(14,0 ; 17,0)$ & 0,237 \\
Faceta 4-Participação Social & $13,0(11,0 ; 16,8)$ & $16,0(15,0 ; 18,0)$ & $0,004 *$ \\
Faceta 5 - Morte e Morrer & $14,5(12,0 ; 18,0)$ & $14,0(12,0 ; 15,8)$ & 0,256 \\
Faceta 6 - Intimidade & $15,5(13,0 ; 17,0)$ & $15,5(12,0 ; 16,0)$ & 0,804 \\
\hline
\end{tabular}

* Diferença significativa: $p<0,05$ - Teste "U" de Mann-Whitney;

Atividades PPF: Atividades passadas, presentes e futuras; G1: idosas não praticantes de exercício físico; G2: idosas usuárias das ATIs; Funcionamento DS: funcionamento dos sentidos

Na comparação da capacidade funcional entre os dois grupos (Tabela 3 ) foi encontrada diferença significativa apenas no teste LCMC $(p=0,001)$ e VTC $(p=$ $0,001)$. Destaca-se que as idosas do $\mathrm{G} 2$ realizaram o teste de LCMC em menor tempo, enquanto as idosas do G1 fizeram em menor tempo o teste de VTC.
Tabela 3 - Comparação da capacidade funcional das idosas usuárias das ATls e das não praticantes de exercício físico

\begin{tabular}{|c|c|c|c|}
\hline \multirow{2}{*}{ VARIÁVEIS } & G1 & G2 & \multirow{2}{*}{$\boldsymbol{P}$} \\
\hline & Md (Q1; Q3) & Md (Q1; Q3) & \\
\hline $\begin{array}{l}\text { Caminhada de } \\
10 \text { minutos }\end{array}$ & $10,7(9,0 ; 13,0)$ & $9,4(6,8 ; 13,0)$ & 0,126 \\
\hline LPS & $12,7(9,7 ; 15,9)$ & $11,8(9,0 ; 14,0)$ & 0,244 \\
\hline LPDV & $7,6(5,0 ; 9,9)$ & $8,3(6,2 ; 14,3)$ & 0,107 \\
\hline LCMC & $45,5(40,4 ; 53,6)$ & $29,3(23,3 ; 32,2)$ & $0,001 *$ \\
\hline VTC & $8,9(6,8 ; 11,8)$ & $13,3(10,2 ; 17,5)$ & $0,001 *$ \\
\hline IG & $31,8(29,2 ; 36,3)$ & $27,7(24,5 ; 36,6)$ & 0,108 \\
\hline
\end{tabular}

* Diferença significativa: $p<0,05$ - Teste "U" de Mann-Whitney; Q1; Q3: quartis.

ATIs: Academias da Terceira idade; G1: idosas não praticantes de exercício físico; G2: idosas usuárias das ATIs. LPS: levantar da posição sentada; LPDV: levantar da posição de decúbito ventral; LCMC: levantar da cadeira e mover-se pela casa; VTC: vestir e tirar a camiseta. IG: Índice GDLAM de capacidade funcional; Md: mediana.

Ao analisar a correlação entre os testes de capacidade funcional e os domínios e facetas de qualidade devida (Tabela 4), foram encontradas correlações significativas $(p<0,05)$ : C10m com domínios Físico $(r=$ $-0,42)$ e Psicológico $(r=-0,29)$; e facetas de Funcionamento dos Sentidos $(r=-0,36)$ e Intimidade $(r=-0,24)$; LPS com a faceta de Funcionamento dos Sentidos ( $r$ $=-0,23)$; LPDV com as facetas de Funcionamento dos Sentidos $(r=-0,23)$ e Atividades Passadas, Presentes e Futuras $(r=0,25)$; LCMC com a faceta de Participação Social $(r=-0,25)$; VTC com a faceta de Funcionamento dos Sentidos ( $r=-0,25)$; e IG com o Domínio Físico ( $r$ $=-0,31)$ e a faceta de Funcionamento dos Sentidos $(r$ $=-0,30)$.

\section{DISCUSSÃO}

Os principais resultados de presente estudo referem-se aos melhores índices de correlação entre a capacidade funcional e alguns aspectos da qualidade de vida de sujeitos idosos do sexo feminino. Além disso, os resultados, embora sem diferenças significativas, mostram que idosas usuárias das ATIs tinham melhores escores de capacidade funciona, quando comparadas com seus pares que não praticavam exercício físico.

Observando as "Facetas de Qualidade de vida" (WHOQOL Old) verificou-se que na faceta de Participação Social foi encontrada uma diferença significante, o grupo G2 apresentou maiores valores quando comparado com o grupo G1. Esse resultado está de acordo com Franco et al. (2015), que mostram em sua revisão que os participantes de exercício sentem-se mais confortáveil sob supervisão profissional 
Tabela 4 - Correlação dos testes de capacidade funcional com os domínios e facetas de qualidade de vida das idosas de ambos os grupos

\begin{tabular}{|c|c|c|c|c|c|c|}
\hline \multirow{2}{*}{$\begin{array}{l}\text { Domínios e Facetas de Qualidade } \\
\text { de vida }\end{array}$} & \multicolumn{6}{|c|}{ Testes de capacidade funcional } \\
\hline & $\mathrm{C} 10 \mathrm{~m}$ & LPS & LPVD & LCMC & VTC & IG \\
\hline D1 - Físico & $-0,42^{*}$ & $-0,20$ & $-0,18$ & $-0,10$ & $-0,10$ & $-0,31 *$ \\
\hline D2 - Psicológico & $-0,29 *$ & $-0,11$ & $-0,02$ & $-0,12$ & $-0,01$ & $-0,18$ \\
\hline D3 - Relações sociais & $-0,19$ & $-0,14$ & 0,09 & $-0,03$ & 0,16 & $-0,03$ \\
\hline D4 - Meio ambiente & $-0,19$ & 0,04 & 0,13 & $-0,03$ & $-0,02$ & $-0,07$ \\
\hline D5 - Autoavaliação & $-0,28 *$ & $-0,13$ & $-0,01$ & 0,07 & $-0,07$ & $-0,14$ \\
\hline F1 - Func. dos Sentidos & $-0,36 *$ & $-0,23 *$ & $-0,23 *$ & 0,13 & $-0,25 *$ & $-0,30 *$ \\
\hline F2 - Autonomia & $-0,12$ & $-0,11$ & $-0,08$ & 0,12 & $-0,04$ & $-0,02$ \\
\hline F3 - Atividades & $-0,08$ & $-0,17$ & $0,25^{*}$ & $-0,14$ & 0,18 & $-0,01$ \\
\hline F4 -Participação Social & $-0,25^{*}$ & $-0,15$ & 0,11 & $-0,25^{*}$ & 0,20 & $-0,11$ \\
\hline F5 - Morte e Morrer & $-0,17$ & $-0,01$ & 0,01 & 0,14 & $-0,05$ & $-0,03$ \\
\hline F6 - Intimidade & $-0,24^{*}$ & $-0,15$ & $-0,12$ & $-0,03$ & $-0,15$ & $-0,15$ \\
\hline
\end{tabular}

*Correlação significativa $(p<0,05)$ - Correlação de Spearman.

e em companhio de pessoas com idade semelhante. Curiosamente, a interação social com os profissionais e pessoas da mesma faixa etári, favorece uma maior percepção nesta variável (FRANCO et al., 2015; SMITH et al., 2017).

Em mais uma análise, agora observando os "Domínios de Qualidade de Vida" (WHOQOL Bref), não foi encontrado diferença entre os grupos, em relação aos domínios avaliados. Embora nenhuma diferença estatística tenha sido encontrada, pode-se observar que para o Domínio Psicológico e Domínio Relações Pessoais foram encontrados maiores escores no grupo G2. Resultado semelhante ao encontrado por Moraes, Correa e Coelho (2018), os quais acharam maior correlação entre os fatores psicológicos e sociais na predição da qualidade de vida. favorecendosa hipótese de que esse maior contato com outras pessoas no mesmo grupo social pode ser benéfico para ter uma melhor percepção da qualidade de vida, como já visto em outros estudos (SOUTO et al., 2016; MORAES; CORREA; COELHO, 2018).

Na Tabela 3 estão apresentados os resultados dos testes funcionais. Os resultados mostram que para o teste de LCMC o grupo $\mathrm{G} 2$ obteve melhores escores que o grupo G1. Esse resultado nos revela que idosas que praticavam exercícios nas ATls possuíam uma melhor capacidade funcional em levantar-se de uma cadeira e mover-se por uma certa distância. Oliveira et al. (2017) obtiveram resultados semelhantes ao comparar idosas praticantes de hidroginástica com não praticantes. Desta forma a prática de exercício físico deve ser estimulada para essa população devido à importância dessa variável para realizações das ABVD e AIVD (SOUTO et al., 2016)
Já no teste VTC, as idosas do G1 obtiveram meIhores resultados que o G2, resultado que foi inesperado. Uma possível explicação para tanto pode ser associado ao fato de que os exercícios praticados nas ATIs não influenciaram diretamente nessa tarefa (vestir e tirar camiseta), e assim não gerando adaptações positivas sobre tal atividade. Além disso, o grupo G2 poderim ter idosas com maiores limitações para realizar tal movimento quando comparado com o grupo G1.

Para o restante dos testes não foram encontradas diferenças significativas, o que hipoteticamente aponta para a necessidade de se intensificar e controlar os exercícios das idosas usuárias das ATIs, assim como de inserir, obrigatoriamente, o profissional de Educação Física nestes locais, a fim de prescrever e orientar adequadamente os exercícios a essa população. Além disso, foi observado uma correlação dos diferentes testes de capacidade funcional com os vários aspectos da qualidade de vida. Na Tabela 4 são apresentados esses resultados, e isso reforça a hipótese de que maiores níveis de funcionalidade, mobilidade, independência física podem ser fatores determinantes para se obter um envelhecimento mais saudável, mais ativo. Esses resultados revelam tal situação, em que vários desses aspectos que constituem a qualidade de vida possuem correlação com a capacidade funcional, aqui representada por alguns testes específicos (OLIVEIRA et al., 2017).

Esses resultados são de suma importância para a prescrição de atividades e exercícios físicos para sujeitos idosos, uma vez que ums das principais variáveis que precism ser melhorads nessa população é a capacidade funcional, que por sua vez pode trazer vários 
outros benefícios à saúde desses sujeitos. Nesse sentidm, limitações devem ser destacadas, como o pequeno número de participantes em ambos os grupos; a não identificação da intensidade, volume e frequência de uso das ATIs no grupo G; e a não avaliação do nível de atividade física do G1, variável que pode ter interferido nos resultados não significativos entre os grupos. Os achados deste trabalho, no entanto, apresentam algumas consideráveis limitações que precisam ser abordadas. Em primeiro lugar, não foi analisado a condição de saúde das idosas participantes do G1. Segundo, não se observou parâmetros do treinamento realizado nas ATIs no G2. Assim sendo, futuras investigações devem explorar essas limitações, uma veo que melhores resultados no G2 incentivariam a prática de exercício pe a população idosa.

\section{CONCLUSÃO}

Apesar de o presente estudo não revelar evidências causais sobre o impacto dos exercícios físicos realizados nas ATIs em todos os domínios da capacidade funcional, os melhores escores obtidos no item levantar-se da cadeira e mover-se pela cas, indicam importantes contribuições possíveis das ATls para a dimensão física das condições de saúde dos idosos, além do benefício na socialização verificada na percepção da qualidade de vida dessa população.

\section{REFERÊNCIAS}

AGOSTINI, C. M. et al. Análise do desempenho motor e do equilíbrio corporal de idosos ativos com hipertensão arterial e diabetes tipo 2. Revista de Atenção à Saúde, v. 16, n. 55, p. 29-35, 2018.

BARBER, S. E.; FORSTER, A.; BIRCH, K. M. Levels and patterns of daily physical activity and sedentary behavior measured objectively in older care home residents in the United Kingdom. journal of Aging and Physical Activity, v. 23, n. 1, p. 133-143, 2015.

BRUCKI, S. M. D. et al. Sugestões para o uso do mini-exame do estado mental no Brasil. Arquivos de Neuropsiquiatria, v. 61, n. 3B, p. 777-781, 2003.

CHUNG, P. et al. A canonical correlation analysis on the relationship between functional fitness and health-related quality of life in older adults. Archives of gerontology and geriatrics, v. 68, p. 44-48, 2017.

DANTAS, E. H. M.; VALE, R. G. S. Protocolo GDLAM de avaliação da autonomia funcional. Fitness \& Performance Journal, v. 3, n. 3, p. 175-182, 2004.

FARÍAS-ANTÚNEZ, S. et al. Incapacidade funcional para atividades básicas e instrumentais da vida diária: um estudo de base populacional com idosos de Pelotas, Rio Grande do Sul, 2014. Epidemiologia e Serviços de Saúde, v. 27, p. e2017290, 2018.
FLECK, M. P.; CHACHAMOVICH, E.; TRENTINI, C. Desenvolvimento e validação da versão em Português do módulo WHOQOL-OLD. Revista de Saúde Pública, v. 40, n. 5, p. 785791, 2006.

FLECK, M. et al. Aplicação da versão em português do instrumento abreviado de avaliação da qualidade de vid" WHOQOL-bre". Revista de Saúde Pública, v. 34, p. 178-183, 2000.

FOLSTEIN, M. F.; FOLSTEIN, S. E.; MCHUGH, P. R. “Mini-mental state": a practical method for grading the cognitive state of patients for the clinician. Journal of Psychiatric Research, v. 12, n. 3, p. 189-198, 1975.

FRANCO, M. R. et al. Older people's perspectives on participation in physical activity: a systematic review and thematic synthesis of qualitative literature. Brazilian Journal of Sports Medicine, v. 49, n. 19, p. 1.268-1.276, 2015.

FREITAS, C. V. et al. Avaliação de fragilidade, capacidade funcional e qualidade de vida dos idosos atendidos no ambulatório de geriatria de um hospital universitário. Revista Brasileira de Geriatria e Gerontologia, v. 19, n. 1, p. 119$128,2016 \ldots . .$.

GONÇALVES, F. B. et al. Qualidade de vida e indicativos de depressão em idosas praticantes de exercícios físicos em academias da terceira idade da cidade de Maringá (PR). Saúde e Pesquisa, v. 8, n. 3, p. 557-567, 2015.

GUTIÉRREZ, M.; CALATAYUD, P.; TOMÁS, J. .. Motives to practice exercise in old age and successful aging: A latent class analysis. Archives of Gerontology and Geriatrics, v. 77, p. 44-50, 2018.

MORAES, F. L. R.; CORRÊA, P.; COELHO, W. S. Avaliação da autonomia funcional, capacidades físicas e qualidade de vida de idosos fisicamente ativos e sedentários. Revista Brasileira de Prescrição e Fisiologia do Exercício (RBPFEX), v. 12, n. 74, p. 297-307, 2018.

MÜLLER, A. M. et al. Physical activity and aging research: a bibliometric analysis. Journal of Aging and Physical Activity, v. 24, n. 3, p. 476-483, 2016.

OMS. Organización Mundial de la Salu). Informe mundial sobre el envejecimiento y la salud. 2015. Disponible en: http://apps.who.int/iris/bitstre am/10665/186466/1/9789240694873_spa.pdf?ua=1.

OLIVEIRA, D. V et al. Capacidade funcional e qualidade de vida em mulheres idosas praticantes e não praticantes de hidroginástica. Revista da Rede de Enfermagem do Nordeste, v. 18, n. 2, p. 156-163, 2017.

PINTO, J. M.; FONTAINE, A. N.; NERI, A. L. The influence of physical and mental health on life satisfaction is mediated by self-rated health: A study with Brazilian elderly. Archives of Gerontology and Geriatrics, v. 65, p. 104-110, 2016.

RIBEIRO, A. S. et al. Effect of resistance training on C-reactive protein, blood glucose and lipid profile in older women with differing levels of RT experience. Age, v. 37, n. 6, p. 109, 2015.

SANTOS, L. et al. Changes in phase angle and body composition induced by resistance training in older women. European Journal of Clinical Nutrition, v. 70, n. 12, p. 1408, 2016. 
SILVA, A. N. C. et al. Fatores motivacionais relacionados à prática de atividades físicas em idosos Psicologia em Estudo, v. 21, n. 4, p. 677-685, 2016.

SIQUEIRA, A. F. et al. Efeito de um programa de fisioterapia aquática no equilíbrio e capacidade funcional de idosos Saúde e Pesquisa, v. 10, n. 2, p. 331-338, 2017.

SMITH, G. L. et al. The association between social support and physical activity in older adults: a systematic review. International Journal of Behavioral Nutrition and Physical Activity, v. 14, n. 1, p. 56, 2017.

SOUTO, S. V. D. et al. Body image in adult vs. middle-aged and elderly women practitioners and non-practitioners of hydro gymnastics/Imagem corporal em mulheres adultas vs. meia-idade e idosas praticantes e nao praticantes de hidroginastica. Motricidade, v. 12, n. 1, p. 53-60, 2016.
VALE, R. G. S. et al. Teste de autonomia funcional: vestir e tirar uma camiseta (VTC). Revista Brasileira de Ciência e Movimento, v. 14, n. 3, p. 71-78, 2008.

VAN ALPHEN, H. J. M.; HORTOBAGYI, T.; VAN HEUVELEN, M. J. G. Barriers, motivators, and facilitators of physical activity in dementia patients: A systematic review. Archives of Gerontology and Geriatrics, v. 66, p. 109-118, 2016. 